\title{
Denkfehler bei diagnostischen Entscheidungen
}

\author{
Martin Gäbler (D)
}

Eingegangen: 10. August 2016 / Angenommen: 8. Mai 2017 / Online publiziert: 23. Mai 2017

(c) Der/die Autor(en) 2017. Dieser Artikel ist eine Open-Access-Publikation.

Zusammenfassung Geschätzte $10-15 \%$ unserer Diagnosen sind falsch und können $\mathrm{zu}$ abwendbaren, gefährlichen Krankheitsverläufen führen. Für einen Großteil dieser Fehldiagnosen sind Denkfehler im diagnostischen Prozess verantwortlich.

Unsere medizinisch-diagnostischen Entscheidungen basieren auf einem intuitivem „System 1“ (Blickdiagnose) und einem analytischem „System 2“ (Differentialdiagnose), welche durch unbewusste Faktoren (Bias) beeinflusst werden und zu Denkfehlern führen. Solche kognitiven Verzerrungen können auf Systemebene und Individualebene positiv beeinflusst werden. Dem Einzelnen helfen im diagnostischen Prozess Metakognition (inneres Zurücktreten vom Entscheidungsprozess) und Debiasingstrategien, wie Verifikation, Falsifikation und Ausschluss möglicher gefährlicher Verlaufsformen, zu besseren diagnostischen Entscheidungen.

Schlüsselwörter Medizinische Entscheidungen · Differentialdiagnose $\cdot$ Fehldiagnose $\cdot$ Blickdiagnose $\cdot$ Metakognition

\section{Cognitive errors in diagnostic decision making}

Summary Approximately $10-15 \%$ of our diagnostic decisions are faulty and may lead to unfavorable and dangerous outcomes, which could be avoided. These

\footnotetext{
$\mathrm{Zu}$ Gunsten der besseren Lesbarkeit wurde für die männliche und die weibliche Form nur eine dieser beiden Formen verwendet.

Dr. med. univ. M. Gäbler, MSc ( $\varangle)$

Institut für Präventiv- und Angewandte Sportmedizin -

Universitätsklinikum Krems, Karl Landsteiner

Privatuniversität für Gesundheitswissenschaften,

Mitterweg 10, 3500 Krems an der Donau, Österreich

gaebler@richtiggesund.at
}

diagnostic errors are mainly caused by cognitive biases in the diagnostic reasoning process.

Our medical diagnostic decision-making is based on intuitive "System 1" and analytical "System 2" diagnostic decision-making and can be deviated by unconscious cognitive biases.

These deviations can be positively influenced on a systemic and an individual level. For the individual, metacognition (internal withdrawal from the decision-making process) and debiasing strategies, such as verification, falsification and rule out worst-case scenarios, can lead to improved diagnostic decisions making.

Keywords Medical Decision Making - Differential Diagnosis · Diagnostic Error · Bias · Metacognition

\section{Einleitung}

Jeder Arzt möchte nach Möglichkeit richtige Diagnosen stellen, doch leider scheint das nicht immer so zu gelingen, wie es wünschenswert wäre [1-4]. Diagnostische Fehlentscheidungen wirken sich auf die Gesundheit, die Lebensqualität und Lebenslänge der Patienten, sowie mit den zunehmenden therapeutischen Möglichkeiten auch auf die Gesamtbehandlungskosten aus. Letzteres v. a. durch die zumeist hohen Kosten einer zu spät begonnenen korrekten Therapie [3, 4]. Es sind sehr oft unbewusste Denkfehler im diagnostischen Prozess, die unsere Fähigkeit, eine richtige Diagnose zu stellen, beeinflussen [5]. Im Folgenden soll auf die Hintergründe von Diagnosefehlern eingegangen werden und der diagnostische Prozess, mögliche Denkfehler und Beeinflussungen durch Bias, sowie Vermeidungsstrategien (v. a. auf individueller Ebene), erläutert werden. 


\section{Hintergrund}

Das Stellen der Diagnose nimmt eine zentrale Stellung im ärztlichen Tun ein, da durch die Klassifizierung und Benennung der Krankheit, eine Aussage über das zugrundeliegende Problem getroffen wird und damit auch schon oft die Weichen für den einzuschlagenden therapeutischen Weg gestellt werden $[1,6]$. Leider ist es oft gar nicht möglich, eine exakte Diagnose zu stellen. In der Allgemeinpraxis kommt es nur in ca. $50 \%$ der Fälle zu einer Diagnose bzw. zu einer Klassifizierung von Krankheitsbildern, bei den anderen $50 \%$ sind es nur beschreibende Symptomgruppen und Einzelsymptome, wie z. B. Fieber und Halsschmerzen [3, $6]$.

Die Ausführlichkeit der Diagnostik richtet sich nach den Notwendigkeiten, Möglichkeiten und Zielsetzungen der Situation. So reicht im Notfall das Symptom des Atem- bzw. Kreislaufstillstandes um mit einer Reanimation zu beginnen. Das primäre Ziel ist, den drohenden Schaden abzuwenden und in weiterer Folge reversible Ursachen zu behandeln. Erst nach Wiederherstellung des spontanen Kreislaufs und der primären Stabilisierung wird versucht, eine (er)klärende Diagnose zu stellen, die zur definitiven Therapie führt [7].

Je nach Studie sind geschätzte $10-15 \%$ unserer Diagnosen falsch [8]. Wobei als Diagnosefehler eine unterlassene, verzögerte oder falsche Diagnosestellung bezeichnet wird, ebenso wie das Unterlassen angebrachter Untersuchungen, Anordnung von nichtindizierten Untersuchungen und Tests, sowie dem Versagen auf Befunde adäquat zu reagieren [2, 9-11].

Die Rate an Fehldiagnosen ist in der Radiologie und der Pathologie mit 2-5\% am niedrigsten und in der klinischen Medizin, und hier der Notfallmedizin, der Allgemeinmedizin und der Inneren Medizin, mit bis zu $15 \%$ am höchsten $[8,12]$. Die am häufigsten übersehene Diagnose mit fatalem Ausgang ist die Pulmonalembolie, die in bis zu $55 \%$ der Fälle erst in der Autopsie erkannt wird $[8,13]$.

Es wird geschätzt, dass in den USA Fehldiagnosen für den Tod von $10 \%$ der Patienten verantwortlich sind [2]. Tehrani et al. [14] konnten zeigen, dass Diagnosefehler (mit knapp $30 \%$ ) der häufigste Grund für Kunstfehlerprozesse in einem Zeitraum von 25 Jahren in den USA waren. In Deutschland waren 2015 knapp $19 \%$ der 12.000 vermuteten Arzthaftungsfälle (die von den Gutachterkommissionen und Schlichtungsstellen der deutschen Ärztekammern behandelt wurden) Diagnose-assoziiert [15].

Zwaan et al. [5] konnten in einer Studie bei $66 \%$ der untersuchten Diagnosen einen Fehler im Diagnoseprozess nachweisen, wobei es in $13,8 \%$ aller Fälle zu einer Fehldiagnose kam, die bei 11,3\% (aller Patienten) einen Schaden zur Folge hatte. Als Schaden wurde dabei jeder Nachteil für den Patienten gewertet, der zu einem verlängerten oder intensiviertem Aufenthalt führte oder eine körperliche Beeinträchtigung (inkl. Tod) zur Folge hatte. Bei $4 \%$ der Patienten wurde allerdings eine falsche Diagnose gestellt, ohne dass ein Fehler im diagnostischen Prozess erkennbar war. Die häufigsten Fehler im diagnostischen Prozess, die $\mathrm{zu}$ einem Schaden führten, standen im Zusammenhang mit der Anordnung oder Interpretation von Labortests.

Prinzipiell geht man davon aus, dass fehlerhafte Diagnosen in $28 \%$ der Fälle durch kognitive Irrtümer, in $19 \%$ systembedingt und in $46 \%$ system- und kognitionsbedingt entstehen [16].

Weniger als $3 \%$ der medizinischen Fehldiagnosen passieren aber durch mangelndes Wissen. Es sind in $14 \%$ Fehler in der Datenerhebung, in $50 \%$ mangelhafte Integration der vorhandenen Informationen und in $33 \%$ Fehler in der Datenverifikation, die zu falschen Diagnosen führen. Letzteres v. a. durch eine vorschnell gestellte Diagnose (Premature Closure) und dem Nichtanordnen von angebrachten Tests (um eine Diagnose zu bestätigen oder auszuschließen), bzw. dem Versäumnis, auf vorliegende Untersuchungsergebnisse zu reagieren [16-18].

Seit dem im Jahr 2000 erschienen Landmark-Report „To err is human: Building a Safer Health System“ [19], ist in der Medizinwelt das Thema Patientensicherheit in den Vordergrund gerückt und hat vielerorts zu einem Umdenken geführt [2, 20]. Allerdings wurde in diesem Report wenig Augenmerk auf Fehler in der Diagnosestellung gelegt [2]. Von der WHOArbeitsgruppe für Patientensicherheit wurde das Thema „Diagnosefehler“ als Forschungsschwerpunkt für 2008 gewählt [21]. 2015 erschien nun der Report „Improving Diagnosis in Health Care"[2], indem das kritische Thema der Diagnosefehler sehr ausführlich behandelt wird. Versuche, diagnostische Fehler zu messen und zugrunde liegende Ursachen zu analysieren, halten sich dennoch in überschaubaren Grenzen. Es wird u. a. vermutet, dass ein mangelndes Verständnis von Entscheidungsbias, das Vermeiden von Diskussionen über Fehldiagnosen und die Schwierigkeiten, solche Fehler zu definieren und zu identifizieren, wesentliche Gründe für diesen Mangel an Beachtung für das Thema sind $[2,4,11,21]$.

\section{Der Diagnoseprozess}

Im klinischen Alltag unterscheiden wir üblicherweise zwischen der Blickdiagnose und der Differentialdiagnose (Abb. 1). Unter Blickdiagnose versteht man die unbewusste, intuitive Diagnosestellung, durch das Erkennen von Mustern, sogenannten Heuristiken. Die Differentialdiagnose hingegen ist das bewusste, analytische Abwägen der klinischen Symptomatik, der vorhandenen Befunde und der Krankheitswahrscheinlichkeiten mit der Bildung von Verdachtsdiagnosen, die im weiteren Verlauf entweder bestätigt oder verworfen werden $[2,18,22]$. Psychologen nennen dieses intuitive Entscheiden der Blickdiagnose „System 1“ bzw. „Schnelles Denken“ und das analytische dif- 
Klinische Fallpräsentation

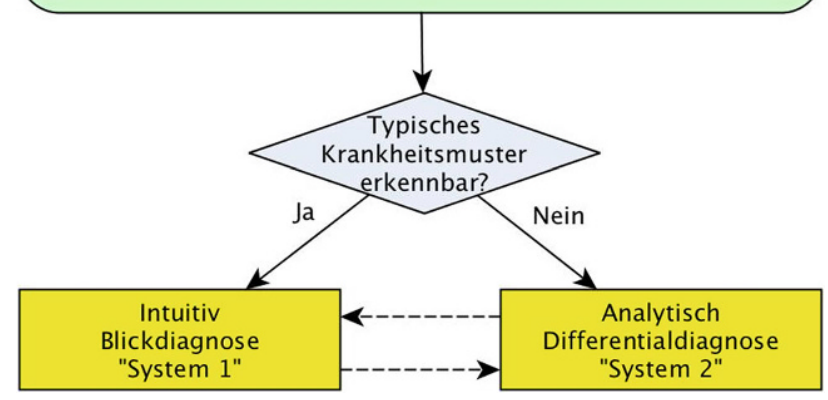

Abb. 1 Diagnosefindung durch eine intuitiv-spontane Blickdiagnose oder analytisch-logische Differentialdiagnose. Grafik nach $[2,18,30]$

ferentialdiagnostische Überlegen „System 2“ bzw. „Langsames Denken“. Diese Denk- und Entscheidungsmechanismen ziehen sich durch viele Bereiche in unserem Leben $[1,20]$.

\section{System 1 - Intuition und Blickdiagnose}

Im intuitiven diagnostischen Entscheiden (System 1 Schnelles Denken) spielt unsere Erfahrung eine große Rolle, die mit wenigen Informationen ein Muster erkennt und fast reflexartig eine Diagnose stellt. Informationstheoretisch spricht man bei dieser Mustererkennung von einer Heuristik, die im Normalfall mit hoher Wahrscheinlichkeit rasch zur richtigen Diagnose führt. Diese Mustererkennung funktioniert bei typischen und häufigen Krankheitsbildern sehr gut, ist aber bei unbekannten oder ungewöhnlichen Situationen stark fehleranfällig für unbewusste Störeinflüsse bzw. Verzerrungen in unserem Denken, einem sogenannten kognitiven Bias [2, 22-25].

Beispiele für kognitive Verzerrungen (Bias) sind in Tab. 1 zusammengestellt.

\section{System 2 - Rationale Analyse und Differentialdiagnose}

Das logisch-rationale bzw. differentialdiagnostische Überlegen (System 2 - langsames Denken) findet vor allem bei unklaren, unbekannten bzw. komplexen Problemen oder bei fehlender Erfahrung seine Anwendung. Man geht von der Prämisse aus, dass man unter Abwägen aller verfügbaren und möglichen Informationen zur besten Entscheidung kommt. Allerdings $\mathrm{zu}$ dem Preis des hohen (zeitlichen und materiellen) Aufwandes und der Begrenzung, dass es niemals möglich ist, „allwissend“ eine Entscheidung zu treffen [2, 22, 24].

Viele Diagnosen können initial nur mit einer bestimmten Wahrscheinlichkeit gestellt oder ausgeschlossen werden und es bleibt oft eine statistische Unsicherheit bestehen, wie in Abb. 2 dargestellt wird $[2,26]$.
Analytisch betrachtet beginnt dieser Diagnoseprozess (Diagnostic Reasoning) bei der Anamneseerhebung und der klinischen Untersuchung, wo aufgrund der gefundenen Hinweise („Cue acquisition“) Verdachtsdiagnosen gebildet werden („Hypothesis generation“). In diese Überlegungen fließen differentialdiagnostisch dazu gehörende, statistisch häufig auftretende, Erkrankungen mit ein. Ebenso wie bedrohliche Diagnosen, deren Übersehen schwere Folgen für den Patienten nach sich ziehen können. Letztere werden auch als „Abwendbare gefährliche Verläufe“ oder „Rule out worst-case scenarios“ (ROWS) bezeichnet. Die Interpretation weiterer diagnostischer Hinweise aus Anamnese, körperlicher Untersuchung und angeordneten Tests helfen, die diagnostischen Hypothesen zu bestätigen oder auszuschließen („Cue interpretation"). Die verbleibende hypothetische Diagnose wird nun evaluiert, was den Prozess der Verifikation und Falsifikation einschließt („Hypothesis evaluation“) [2, $3,6,18,22,26,27]$. Wenn sich in diesem Diagnoseprozess Widersprüchlichkeiten ergeben oder die Diagnose nicht gestellt werden kann, werden (zumeist automatisch) einer oder mehrerer dieser Schritte wiederholt [2].

Abb. 3 stellt den Diagnoseprozess grafisch dar.

\section{Dualer Entscheidungsprozess im Alltag}

Die Trennung zwischen den beiden Systemen besteht jedoch nur in der Theorie. Wenn wir ein (Krankheits-) Muster erkennen, entscheiden wir im Normalfall nach „System 1“, also intuitiv. Erkennen wir es nicht oder finden sich nach initialer Blickdiagnose Fakten, die nicht passen (sofern wir nicht einem kognitiven Bias erliegen und diese Fakten negieren), wechseln wir in das „System 2“ und arbeiten - aufbauend auf Leitsymptome oder Befunde - unsere differentialdiagnostischen Hypothesen ab, wobei wir versuchen, schwerwiegende (bedrohliche) Diagnosen möglichst auszuschließen [3, 22, 28]. Zumeist laufen aber beide Denkarten parallel ab, was auch als „Dual Process Theory“ bezeichnet wird (Abb. 4, [2, 29]). Der abschließende Schritt in der Diagnosestellung ist die Überprüfung der Arbeitsdiagnose (Hypothesis Evalution). Einerseits, ob sie schlüssig mit den vorhandenen und neu hinzugekommenen Befunden übereinstimmt (Verifikation), andererseits aber, ob nicht doch eine andere Diagnose wahrscheinlicher ist (Falsifikation) $[26,30]$.

\section{Kognitive Verzerrungen - Bias}

Kognitive Verzerrungen sind unbewusste Störeinflüsse im Denkprozess, die als kognitiver Bias bezeichnet werden. Diese Verzerrungen finden sich bei beiden diagnostischen Prozessen, wobei der Bereich der Blickdiagnose - System 1 stärker davon betroffen ist [2, 22].

Eine repräsentative Liste dieser Bias mit Erklärungen sind in Tab. 1 zusammengestellt. 
Abb. 2 Diagnosewahrscheinlichkeitals Kontinuum zwischen Krankheitsausschluss und -bestätigung. Grafik nach [26, 55]

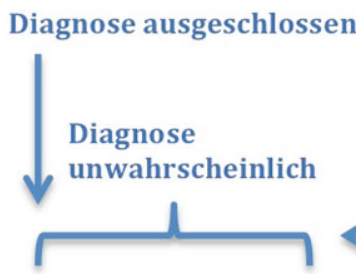

Diagnose gesichert

0

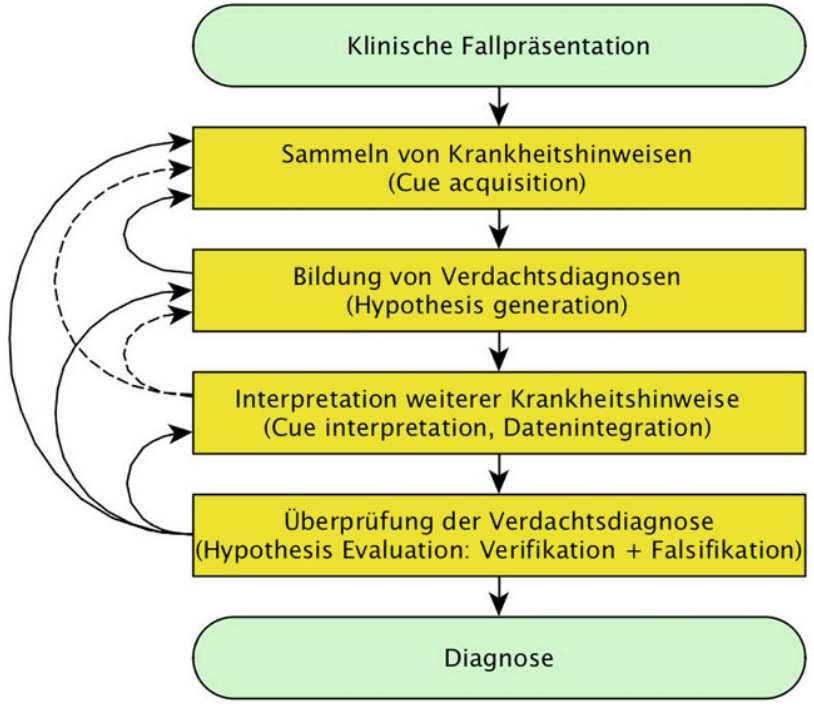

Abb. 3 Analytisch-deduktiver Prozess der Diagnosefindung (Diagnostic Reasoning). Grafik nach [2, 26]

Bestimmte medizinische Bereiche dürften unterschiedlich stark von verschiedenen Bias betroffen sein, je nach Patientenkollektiv und Begleitumstände, wobei es dazu noch keine publizierten validen Untersuchungen gibt. So liegt es nahe, dass es in Bereichen mit hohem Patientenaufkommen und Zeitdruck, wo eine rasche Diagnosefindung im Vordergrund steht (wie z. B. in Notaufnahmen oder Arztpraxen), es leicht zu einem Confirmation Bias bzw. Search Satisficing Premature Closure kommen kann.

\section{Faktoren, die zur Patientensicherheit beitragen}

Verschiedene Gegebenheiten tragen in diesem diagnostischen Prozess zur Patientensicherheit bei. Erhobene Bild-, Labor- und andere Befunde werden im Normalfall auch von einem anderen Mediziner gesehen und es ist (zumindest in den Krankenhäusern, in denen der Autor gearbeitet hat) üblich, bei bedrohlichen Befunden telefonischen Kontakt mit dem behandelnden Arzt bzw. der behandelnden Abteilung aufzunehmen.

Nachdem viele Krankheiten sich erst im zeitlichen Verlauf nach und nach demaskieren und zu Beginn oftmals wichtige Befunde fehlen, ist eine Diagnosestellung nicht immer sofort möglich [11]. So kommt es auch an Notaufnahmen bei nicht-lebensbedrohlichen Erkrankungen fast immer zu einer Wartezeit nach der initialen Diagnostik, in der die Symptomatik klarer wird, aggraviert oder sich bessert, was bei regelmäßiger Evaluation erfasst wird. Auch an der Bettenstation werden die aufgenommenen Patienten im Normalfall noch am gleichen Tag von einem zweiten Arzt gesehen, bzw. seine Befunde in der Aufnahmevisite besprochen. Hier trägt der längere zeitliche Verlauf des stationären Aufenthaltes, mit den verschiedenen an der Behandlung beteiligten Berufsgruppen, nicht unwesentlich zur Diagnose- und Patientensicherheit bei. Somit ist Wartezeit auch eine manchmal notwendige und wertvolle Zeit für die Diagnosefindung.

Bei Entlassung des Patienten stellt die Vidierung des Arztbriefes die letzte Möglichkeit in diesem Prozess dar, die vorhandenen Informationen und Diagnosen zu überprüfen und zu korrigieren.

Auch im niedergelassenen Bereich erfolgt nach der initialen Konsultation bei unklaren oder kritischen Befunden entweder eine Wiederbestellung nach einer bestimmten Zeit oder Patienten, die aufgrund ihrer klinischen Symptomatik schwer krank oder bedroht wirken, werden im Normalfall sofort zu einem Spezialisten oder ins Krankenhaus transferiert. Eine gute Aufklärung des Patienten (bzw. seiner Betreuungsperson) bei der Entlassung, bei welchen Problemen auf alle Fälle erneute ärztliche Hilfe in Anspruch genommen werden soll, hilft Probleme frühzeitig zu erkennen und eventuelle Diagnosefehler zu korrigieren. Damit werden auch die Patienten in die Pflicht genommen, bei Unklarheiten den Kontakt mit dem Behandler zu suchen [2].

Die folgenden klinischen Fallbeispiele sollen Bias in der Diagnostik veranschaulichen.

\section{Fallbeispiel 1}

Bei einem afebrilen, morbid-adipösen, multimorbiden, 86-jährigen Patienten war am Vortag im Rahmen der abendlichen Dialyse eine deutliche CRP-Erhöhung und Leukozytose aufgefallen, weshalb er stationär aufgenommen wurde.

Am nächsten Tag erfolgte der Transfer an die geriatrische Abteilung. Dabei wurde darauf hingewiesen, dass der eigentliche Grund des Infektes noch unklar sei. Allerdings war, unter der Annahme eines neuerli- 


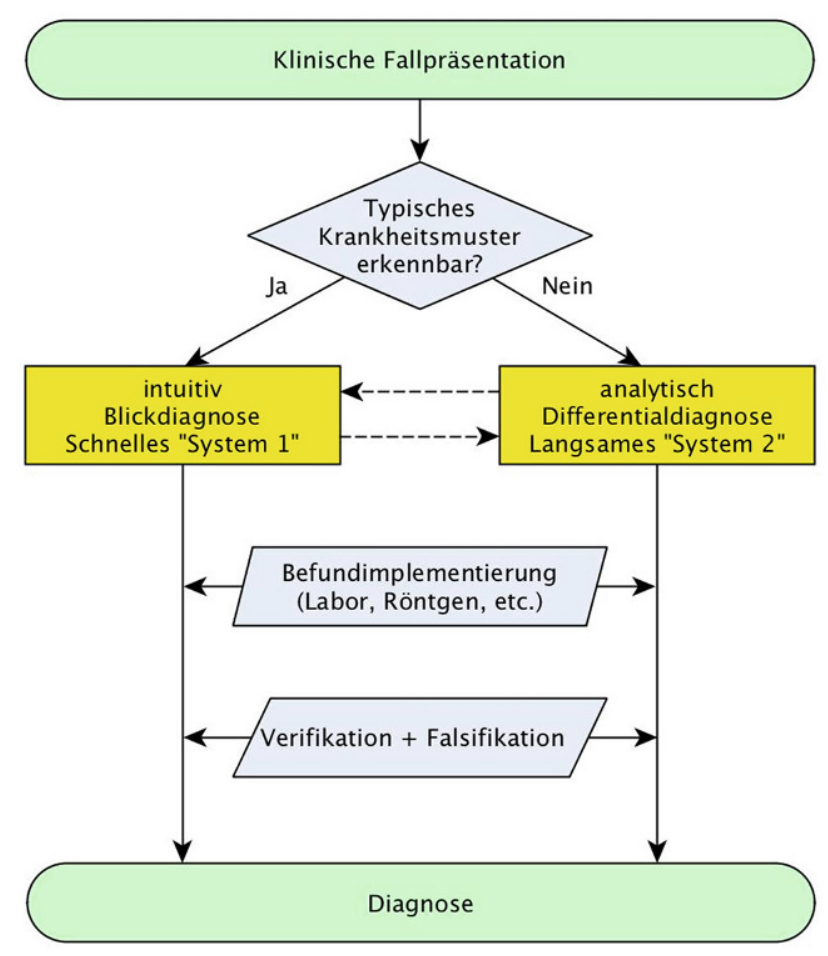

Abb. 4 Gesamtdarstellung des dualen Prozesses der Diagnosefindung im Alltag. Grafik nach [2, 18, 26, 29, 30]

chen Harnwegsinfektes, bereits eine Harnkultur abgenommen und eine empirische antibiotische Therapie eingeleitet worden.

Der Patient war zu diesem Zeitpunkt kardiorespiratorisch stabil und afebril.

An Vorerkrankungen bestanden eine dialysepflichtige diabetische Nephropathie, eine dilatative Kardiomyopathie mit hochgradig eingeschränkter Linksventrikelfunktion, Z.n. Schrittmacherimplantation, Immobilität bei Z.n. multiplen Knie- und Hüftprothesen-Implantationen und -Revisionen (wegen Prothesenlockerungen und einer chronischen Protheseninfektion mit Fistulierung im Kniebereich), sowie rezidivierenden Harnwegsinfektionen und einer Schmerztherapie mit Opioiden unter gleichzeitiger Laxantiengabe. Außerdem war eine renale Anämie und eine chronische CRP Erhöhung mit Werten um $10-12 \mathrm{mg} / \mathrm{dl}$ bekannt. Viele Komplikationen, wie eine interprothetische Oberschenkelfraktur und septische Zustandsbilder, hatten zu regelmäßigen stationären Aufenthalten geführt.

In der neuerlichen Anamnese berichtete der Patient nun über eine seit mehr als einer Woche bestehende Obstipation. Diese hätte sich trotz Bauch- und Rückenmassagen und Gabe abführender Mittel nicht gebessert.

In der klinischen Untersuchung fanden sich keine Auffälligkeiten im Herz-Lungenbereich. Jedoch bestand bei weit ausladendem Abdomen eine diskrete Defense mit Druckschmerz im rechten Mittel- und Unterbauch. Auf neuerliches Befragen gab der Pati- ent an, drei Tage vor Aufnahme einmalig Schüttelfrost gehabt zu haben. Der hausärztliche Notdienst sei geholt worden, habe aber nichts gefunden und habe den Patienten zuhause belassen. Es sei zu keiner weiteren Beschwerdezunahme gekommen.

Im CT des Abdomens findet sich nun freie Luft im Bauchraum und es wird mit hoher Wahrscheinlichkeit eine Darmperforation suspiziert.

Der Patient entscheidet sich, trotz des hohen OPRisikos, für die Operation, wo eine Perforation der rechten Colonflexur mit Peritonitis gefunden wird. Im postoperativen Verlauf kommt es allerdings zu einem Multiorganversagen, welches der Patient - bei fehlender Organreserve - nicht überlebt.

\section{Diskussion - Fallbeispiel 1}

Schwere Erkrankungen bei alten Patienten stellen sich sehr oft symptomarm dar und können durch eine gleichzeitige Schmerztherapie, wie bei unserem Patienten, kaschiert werden. Leider wurde dadurch die vermutlich bereits Tage vor der Aufnahme erfolgte Perforation nicht erkannt. Wie weit die Adipositas hier zu einem Viszeral Bias geführt hat, war nicht eruierbar.

Dass der Patient bei der regelmäßigen Dialyse als behandlungsbedürftig identifiziert und (gegen seinen anfänglichen Widerstand) stationär aufgenommen wurde, war retrospektiv eine richtige Entscheidung.

Die initiale Verdachtsdiagnose, dass ein neuerlicher Harnwegsinfekt bestand, war ein Posterior Probability Error, der den Kollegen aufgefallen ist und auf den bei der Übergabe an die weiterbehandelnde geriatrische Abteilung hingewiesen wurde.

Trotz des sehr hohen OP-Risikos und des unsicheren Ausganges hatte sich der Patient bewusst für die Operation entschieden, weil er diese (kleine) Überlebenschance nutzen wollte. Prinzipiell hätte er diese Option auch ablehnen können. Er war zum Zeitpunkt der Entscheidung bei ausgezeichnetem kognitiven Zustand, entscheidungs- und handlungsfähig [31-34].

\section{Fallbeispiel 2}

Ein 28jähriger Flüchtling kommt in Begleitung seiner Freunde an einem Vormittag von einem Fitness-Studio in die Notaufnahme. Soweit es trotz der Sprachbarriere zu erfahren ist, klagt er über massive Übelkeit und gibt einen dumpfen Druck in der Brust an, was während seiner Übungen mit Gewichten plötzlich begonnen habe.

Er wirkt nervös-unruhig, fast agitiert, und hyperventiliert leicht.

In der klinischen Untersuchung sind die Wirbelsäule und der Brustkorb druck-indolent, die Herztöne rein, rhythmisch, leicht tachykard und die Lungen frei. Das Abdomen, inkl. Nierenlager, ist ebenfalls unauffällig. Die Haut ist südländisch dunkel pigmentiert. 
Tab. 1 Auflistung von häufigen Bias mit Erklärung

Anchoring Man nimmt eine bestimmte Information wichtiger als sie ist, hält daran fest und übersieht dadurch andere Fakten [2, 22].

Dies hängt manchmal mit der Spezialisierung zusammen, durch die man auf einen bestimmten Bereich fixiert ist. Beispielshaft dafür ist die oft kolportierte Geschichte des Patienten, der mit Oberbauchschmerzen an eine Gastroenterologie kommt und bei dem man erst nach einer unauffälligen Gastroskopie den Myokardinfarkt als eigentliche Ursache entdeckt

Availability Bias Das, womit man sich zuletzt beschäftigt hat, fällt auch als Erstes wieder ein. So kann eine seltene Diagnose plötzlich häufig erscheinen, weil sie präsent ist und zu passen scheint [2].

D. h. wenn man gerade eine erweiterte Abklärung bei Transaminasenerhöhung gemacht und sich mit den Formen der Autoimmun-Hepatitis beschäftigt hat, wird einem diese Differentialdiagnose beim nächsten Patient mit erhöhten Transaminasen eher einfallen

Attribution Error Man schiebt den Grund für eine Beschwerde auf ein Persönlichkeitsmerkmal oder eine Patientengruppe und nimmt deswegen Symptome nicht ernst [22, 49].

Typische Schlagwörter, die dabei (unbewusst) verwendet werden sind, „, wehleidig“, „lästig“, „alkoholkrank“, , ,vegetativ überlagert“ oder "depressiv".

"Der wenig professionelle Ausdruck des „lästigen Patienten“, drückt zwei Dinge aus. Einerseits, dass die Beschäftigung mit diesem Patienten mühsam ist und man andererseits meistens nicht weiß oder versteht, was das Problem ist, weshalb man die Beschwerden einem Charaktermerkmal zuschreibt.

Ein weiterer Attribution Error ist die Nichtbeachtung von Symptomen aufgrund des Alters des Patienten, die dann (manchmal auch berechtigt) von Patienten und Ärzten als „Altersbeschwerden“ hingenommen werden. Wenn jedoch aufgrund des chronologischen Alters (bewusst oder unbewusst) diagnostische oder therapeutische Maßnahmen vorenthalten werden, die ein jüngerer Patient mit der gleichen Präsentation bei identer biopsychosozialer Fittheit bekommen würde, entspricht dies einer, als „Ageism“ bezeichneten Diskriminierung [56-58]

Confirmation Man sucht unbewusst die Fakten, die das eigene Konzept bestätigen. D. h. sobald man glaubt, dass eine bestimmte Diagnose stimmt, sucht

Bias man Fakten, die diese Theorie unterstützen und blendet widersprüchliche Informationen, die nicht zu dieser Theorie passen, aus [22, 49]

Context Error

Symptome werden einem Organsystem zugeordnet und damit andere Ursachen nicht in Betracht gezogen [12].

Dabei werden z. B. nur die Bauchorgane als Ursache für Oberbauchbeschwerden, nicht aber eine mögliche kardiale oder pneumologische Ursache in die differentialdiagnostischen Überlegungen einbezogen

Framing Je nachdem, wie die Information präsentiert wird, wird die Entscheidung davon geleitet [18, 54].

Es macht einen großen Unterschied in den differentialdiagnostischen Überlegungen, ob ein und derselbe Patient mit dem Symptom „Hyperventilation" oder "Belastungsdyspnoe" vorgestellt wird

Overconfidence Das (übertriebene) Vertrauen in die eigenen Fähigkeiten führt im besten Fall zu einer raschen korrekten Diagnose, im schlechtesten Fall verhindert es die Reevaluierung und Korrektur eines einmal eingeschlagenen falschen Weges. Dies betrifft den Anfänger und Erfahrenen gleichermaßen. Wer sich zu sicher ist, wird wichtige Diagnosen übersehen [12]. Ein Schlagwort dazu ist auch der „Overconfident-Learner“, der Anfänger, der glaubt, schon alles zu wissen

Posterior Proba- Eine Krankheit, die schon öfter bei diesem Patienten aufgetreten ist, wird automatisch wieder als Ursache angenommen [49].

bility Error Ein Patient, der mehrfach wegen alkoholbedingter Somnolenz in die Notaufnahme gebracht worden ist, hat bei neuerlicher Aufnahme im somnolenten Zustand ein höheres Risiko, dass der abwendbare gefährliche Verlauf eines intrazerebralen Geschehens nicht ausgeschlossen wird, weil er vorschnell als „wie immer" schwer alkoholisiert, eingestuft wird.

Bei geriatrischen Patienten mit chronischen Erkrankungen, die wiederholt mit der gleichen Symptomatik vorstellig werden, ist die Wahrscheinlichkeit hoch, dass bei jedem neuen Erkrankungs- bzw. Hospitalisierungsfall, die Erstdiagnose übernommen wird, ohne relevante Differentialdiagnosen zu überlegen bzw. auszuschließen

Search Satisfi- Das Finden eines bestätigenden Hinweises oder einer Diagnose, welche die Symptome halbwegs erklärt, führt dazu, dass man vorschnell mit cing/Premature den diagnostischen Überlegungen aufhört [12].

Closure $\quad$ Z. B. wenn man sich beim Symptom „thorakale Schmerzen“ vorzeitig für die Diagnose „Akutes Koronarsyndrom“ entscheidet, ohne andere wichtige Ursachen zu exkludieren.

Dieser Bias der vorschnellen Diagnose findet sich in allen Patientengruppen häufig. Interessanterweise erliegen inm erfahrene und junge Kliniker gleichermaßen, jedoch sind ältere Ärzte stärker gefährdet [16, 59]

Visceral Bias Gute oder schlechte Gefühle gegenüber dem Patienten beeinflussen die Entscheidungen im Sinn einer Übertragung und Gegenübertragung $[18,22]$.

Wenn einem das Gegenüber sympathisch ist, kann es dazu führen, dass man in der Abklärung bestimmte Test nicht macht, die invasiv sind oder eine schwerwiegende Konsequenz haben, aber auch, dass man mehr macht, um alles auszuschließen [22]. Typische negative Beeinflussungen geschehen u. a. durch Adipositas, Lebensstil, Suchterkrankung, psychiatrische Probleme oder ethnische Abstammung

Die Sauerstoffsättigung ist mit $99 \%$ normal, kann allerdings an der rechten Hand nicht suffizient abgeleitet werden. Die Blutgasanalyse erfolgt von der linken A. radialis (die rechte A. radialis ist für die Punktion nicht ausreichend tastbar) und zeigt eine leichte respiratorische Alkalose. Der Troponin-Schnelltest und das EKG sind unauffällig. Der Patient erhält eine Metoclopramid-Infusion gegen die Übelkeit und es wird überlegt, ihn unter dem Verdacht, dass er möglicherweise an einer Angststörung leidet, mit einer nervenberuhigenden Medikation nach Hause zu schicken. Allerdings soll noch das Ergebnis der Blutabnahme abgewartet werden.

Ungefähr eine Stunde später sucht der Patient die Toilette auf, wo er zusammenbricht und einen Kreis- laufstillstand erleidet. Trotz der rasch begonnenen Reanimation kann der Patient nicht gerettet werden.

\section{Diskussion - Fallbeipiel 2}

In diesem tragischen Fall einer Aortendissektion haben eine atypische Fallpräsentation, die Sprachbarriere und möglicherweise mehrere Bias verhindert, dass es rechtzeitig zu einer korrekten Diagnose gekommen ist und man eine Verlegung in ein Zentralkrankenhaus mit Thorax-Chirurgie durchführen hätte können. Ob der junge Mann bei seinem raschen Verlauf zeitgerecht operiert werden hätte können (und so überlebt hätte), bleibt spekulativ. 
Die südländische Ethnie (und die vermutete psychische Traumatisierung) des Kriegsflüchtlings, dürfte zu einem Attribution Error, bzw. Visceral Bias, mit der Verdachtsdiagnose Angststörung, geführt haben. Ebenso wurde die bestehende Seitendifferenz des Radialispulses nicht als diagnostischer Puzzlestein erfasst, was einem Confirmation Bias entspricht. Zusätzlich lässt sich ein Premature Closure/Search Satisficing erkennen, da man sich, scheinbar begünstigt durch den hohen Arbeitsdruck, vorerst für die Verdachtsdiagnose Hyperventilation bei Angststörung, entschieden hatte.

\section{Vermeidung von Denkfehlern bei diagnostischen Entscheidungen}

Die heutigen juristischen und fachlichen Anforderungen und Erwartungen an die Diagnostik einerseits und die zunehmenden ökonomischen Zwänge andererseits, stellen eine große Herausforderung und ein Ressourcenproblem für das System und den einzelnen dar, was durch den bestehenden Ärztemangel noch verschärft wird. Wo früher von Patienten ein abwartendes Beobachten akzeptiert wurde, wird heute eine korrekte Diagnosestellung selbst bei komplexer Differentialdiagnostik, zu jeder Tages- oder Nachtzeit, erwartet.

Daher stellen Strategien zur Verbesserung von diagnostischen Entscheidungen eine notwendige und wichtige Management- und Führungsaufgabe dar, die zu einer besseren Struktur-, Prozess- und Ergebnisqualität führt und kontinuierlich angestrebt werden sollte [2, 25, 35].

Ähnliche Anforderungen an die Zuverlässigkeit und Sicherheit von Prozessen, wie in der Medizin, werden im Bereich der Flugsicherheit, in sicherheitskritischen Industrieprozessen (z. B. in Atomkraftwerken) oder im militärischem Bereich (z. B. auf Flugzeugträgern) gestellt, was bereits seit längerem zu einem Wissensund Methodentransfer aus diesen Bereichen in die Medizin geführt hat [20, 25, 36, 37].

Waeschle et al. [25] beschreiben in Ihrem Artikel „Fehler in der Medizin“ Qualitätsverbesserungsstrategien, deren Implementierung jedoch einer kontinuierlichen Anstrengung im Sinne der Anwendung eines PDCA-Zyklus (Plan-Do-Check-Act nach Deming) bedarf $[20,25]$. Sie spannen dabei den Bogen von den systemischen Schwachstellen bis zur Individualebene und beziehen Themen wie Crew Ressource Management, Critical Incident Reporting Systems, statische und dynamische Checklisten, Prospective Memory, etc., mit ein [25]. Zusätzlich können regelmäßige Fallbesprechungen - im Sinn von „Morbidity and Mortality“ Konferenzen-, wenn sie Teil eines PDCA-Zyklus sind, ebenfalls zur Qualitätsverbesserung beitragen [38, 39]. Hier kann die Verwendung von Fishbone-Diagrammen (auch als Ishikawa-Diagramm oder Root-Cause-Analysis bekannt) helfen, die verschiedenen Ursachen und beeinflussenden Fakto- ren systematisch aufzuarbeiten [2, 40, 41]. Wobei fehlerhafte Entscheidungen oft durch eine Kombination von Ursachen (auf System- und Individualebene) entstehen [36, 42].

Systembedingte Ursachen, die sich auf unser Denken auswirken und sich durchaus korrigieren lassen, sind strukturelle Gegebenheiten wie veraltete bauliche Strukturen, fehlende klinische Standards, Schlafmangel, Arbeitsüberlastung, Personalmangel, ineffiziente Arbeitsprozesse, zu hohe Fehlerakzeptanz, mangelnde Fehlerkultur und fehlendes Feedback [2, 25, 36, 43].

Gerade in komplexen Unternehmen, wie es Krankenhäuser und Notfallaufnahmen sind, lässt sich u. a. durch eine kollektive Änderung in der Sensibilität für mögliche Fehlerquellen eine Verbesserung der Patientensicherheit erzielen, wozu aber eine bewusste Veränderung der Betriebskultur notwendig ist, um von einer „Blame and Shame“ Mentalität zu einem offenen und konstruktiven Umgang mit Fehlern zu kommen, wo Fehler frühzeitig angesprochen und diskutiert werden können [25, 36, 44, 45].

Verbesserungen der individuellen Ursachen und damit des diagnostischen Entscheidens gestalten sich da schwieriger, sind vielschichtiger und benötigen wiederholtes Training und die Anwendung verschiedener Strategien [21, 25]. Nur vom Einzelnen zu verlangen, mehr aufzupassen, bringt leider wenig, da es per se nicht $\mathrm{zu}$ weniger Fehlern führt, wie Berwick [46] bereits 2001 in seinem Artikel „Not again!“ schreibt.

Das Wissen um unbewusste Beeinflussungen (Bias) kann dem einzelnen Arzt helfen, adäquat darauf zu reagieren und Debiasing Strategien zu lernen. Dazu gehören die Wissensvermittlung über den diagnostischen Prozess, Metakognition und Reflexion (im Sinne eines inneren Zurücktretens und Bewertens von Situation, Gedankenprozessen und getroffenen Entscheidungen), sowie des Bewusstmachens von möglichen Bias. Ebenso aber auch zu fragen, ob eine andere Diagnose möglich ist (Falsifikation), ob die erhobenen Fakten mit der Arbeitsdiagnose übereinstimmen (Verifikation) und welche Diagnose nicht übersehen werden darf (im Sinn des abwendbaren gefährlichen Verlaufes) [3, 12, 47, 48]. Dabei helfen Cognitive Forcing-Strategies, wo man sich bei bestimmten häufigen Symptomen zwingt, definierte Diagnosen im Sinn einer mentalen Checkliste auszuschließen, wie z. B. die „dramatischen Big-5“ des akuten thorakalen Schmerzes (ACS, Lungenembolie, Spannungspneumothorax, Aortendissektion, Ösophagusruptur) [49, 50].

Zusätzliche Unterstützung im diagnostischen Prozess erhält man durch die Verwendung von Checklisten, Mnemotechniken (Mnemonics), Algorithmen und elektronischen Unterstützungssystemen [21, 28, 51]. Letztere bieten ein großes Potential an Möglichkeiten und werden den ärztlichen Alltag vermutlich noch mehr beeinflussen, als man es sich derzeit vorstellen kann [2, 52, 53]. Allerdings kann ein schlecht gestaltetes digitales Informationssystem diese Vortei- 
le rasch zunichte machen und ein Patientensicherheitsrisiko darstellen [2]. Elektronische Informationssysteme werden seit Jahren für die Bereitstellung und Weitergabe von Patienteninformationen (z. B. von Befunden, Arztbriefen, elektronischer Gesundheitsakte, etc.) und als Wissensdatenbanken mit Zugriff $\mathrm{zu}$ relevanten Informationen, wie Guidelines, etc., genutzt. Interaktive Systeme für das Work-up von diagnostischen Entscheidungen können durch die Verwendung von Abfrageschemata für Differentialdiagnosen, Erinnerungen bei offenen Befunden und Fragestellungen, automatischen Interpretationen von Befundmustern, Integration von Hinweisen auf Medikamentennebenund -wechselwirkungen, Berechnungen von Krankheitswahrscheinlichkeiten, Rückmeldungen über die Compliance und das wirkliche Outcome, rascheren Zugriff auf Zweitmeinungen, usw., eine Unterstützung im diagnostischen Prozess sein [53].

\section{So What?}

Folgende drei Fragen können uns im Alltag, ungeachtet aller systemischer und struktureller Widrigkeiten, helfen, Denkfehler im diagnostischen Prozess zu erkennen.

1. Angenommen, die Diagnose ist falsch, welche alternative Diagnose kommt in Frage? = Falsifikation, zur Aufdeckung des häufigsten Bias, dem Premature Closure [3, 16].

2. Werden die Beschwerden vollständig durch die Diagnose erklärt? = Verifikation [2].

3. Gibt es eine Diagnose, die man hier nicht übersehen darf? = Ausschluss eines abwendbaren gefährlichen Verlaufes [3, 22].

\section{Schlussbemerkung}

In der Entscheidungswissenschaft, an der Philosophen, Psychologen, Ökonomen und andere forschen, ist sehr viel im Fluss und man kann persönlich für sich, seine Patienten und seine Arbeitszufriedenheit, sehr davon profitieren, wenn man sich konstruktiv damit auseinander setzt. Allerdings ist die Medizin keine theoretische Wissenschaft, sondern eine praktisch handelnde, und so wird man immer gefordert sein, Entscheidungen unter Unsicherheit nach bestem Wissen und Gewissen zu treffen $[1,26]$.

Weiterführende Literatur

- http://smdm.org

- http://www.improvediagnosis.org

- http://qualitysafety.bmj.com

- Balogh, Miller, Ball, Improving Diagnosis in Health Care [2]

- Sox, Medical Decision Making [26]

- Gigerenzer, Bauchentscheidungen [54]

\section{Fazit für die Praxis}

Ein Teil der gestellten Fehldiagnosen entstehen durch Denkfehler in unserem diagnostischen Prozess. Sie können durch inneres Zurücktreten von diesem Prozess und strukturiertem Hinterfragen der eigenen Diagnose identifiziert werden.

\section{Einhaltung ethischer Richtlinien}

Interessenkonflikt M. Gäbler gibt an, dass kein Interessenkonflikt besteht.

Ethische Standards Dieser Beitrag beinhaltet keine vom Autor durchgeführten Studien an Menschen oder Tieren.

Open Access Dieser Artikel wird unter der Creative Commons Namensnennung 4.0 International Lizenz (http:// creativecommons.org/licenses/by/4.0/deed.de) veröffentlicht, welche die Nutzung, Vervielfältigung, Bearbeitung, Verbreitung und Wiedergabe in jeglichem Medium und Format erlaubt, sofern Sie den/die ursprünglichen Autor(en) und die Quelle ordnungsgemäß nennen, einen Link zur Creative Commons Lizenz beifügen und angeben, ob Änderungen vorgenommen wurden.

\section{Literatur}

1. Wieland W. Diagnose: Überlegungen zur Medizintheorie. Bd.2. Warendorf: Hoof; 2015.

2. Balogh EP, Miller BT, Ball JR. et al. Improving Diagnosis in Health Care [Internet]. Washington, DC: The National Academies Press 2015. http://www.nap.edu/catalog/21794/ improving-diagnosis-in-health-care.Zugegriffen: 4. Oktober 2015.

3. Braun RN, Mader FH, Danninger H. Programmierte Diagnostik in der Allgemeinmedizin. Heidelberg Berlin: Springer; 2005.

4. Khullar D, Jha AK, Jena AB. Reducing diagnostic errors-why now? NEngl J Med. 2015;373:2491-3.

5. Zwaan L, Thijs A, Wagner C, van der Wal G, Timmermans DRM. Relating faults in diagnostic reasoning with diagnostic errors and patient harm. Acad Med. 2012;87:149-56.

6. Mader FH. Allgemeinmedizin und Praxis, 7. Aufl. Berlin, Heidelberg: Springer; 2014.

7. Soar J, Nolan JP, Böttiger BW, Perkins GD, Lott C, Carli P, etal.ErweiterteReanimationsmaßnahmenfürErwachsene (,adult advanced life support“): Kapitel 3 der Leitlinien zur Reanimation 2015 des European Resuscitation Council. NotfRettungsmed.2015;18:770-832.

8. Croskerry P. From mindless to mindful practice - cognitive bias and clinical decision making. $\mathrm{N}$ Engl J Med. 2013;368:2445-8.

9. Hölscher UM, Gausmann P, Haindl H, Heidecke C-D, Hübner N-O, Lauer W, et al. Übersichtsartikel: Patientensicherheit als nationales Gesundheitsziel: Status und notwendige Handlungsfelder für die Gesundheitsversorgung in Deutschland. Z Evid Fortbild Qual Gesundheitswes. 2014;108:6-14.

10. Balla J, Heneghan C, Goyder C, Thompson M. Identifying early warning signs for diagnostic errors in primary care: a qualitative study. BMJ Open. 2012;2:e001539.

11. ZwaanL, SinghH. The challengesindefining andmeasuring diagnostic error. Diagnosis 2015. http://www.degruyter. $\mathrm{com} / \mathrm{view} / \mathrm{j} / \mathrm{dx} .2015 .2$.issue-2/dx-2014-0069/dx-20140069.xml. Zugegriffen:23. Febr 2017. 
12. Berner ES, Graber ML. Overconfidence as a cause of diagnostic error in medicine. Am J Med. 2008;121:S2-23.

13. Pineda LA, Hathwar VS, Grant BJ. Clinical suspicion of fatal pulmonary embolism. Chest. 2001;120:791-5.

14. Tehrani ASS, Lee H, Mathews SC, Shore A, Makary MA, Pronovost PJ, et al. 25-Year summary of US malpractice claims for diagnostic errors 1986-2010: an analysis from the National Practitioner Data Bank. BMJ Qual Saf. 2013;22:672-80.

15. Bundesärztekammer. Behandlungsfehler-Statistik der Gutachterkommissionen und Schlichtungsstellen 2015. http:/ / www.bundesaerztekammer.de/patienten/ gutachterkommissionen-schlichtungsstellen/ behandlungsfehler-statistik-2015/.Zugegriffen: 23. Febr 2017.

16. Graber ML, Franklin N, Gordon R. Diagnostic error in internal medicine. Arch Intern Med. 2005;165:1493-9.

17. Bordage G. Why did I miss the diagnosis? Some cognitive explanations and educational implications. Acad Med. 1999;74:S138-S43.

18. Nendaz M, Perrier A. Diagnostic errors and flaws in clinical reasoning: mechanisms and prevention in practice. Swiss MedWkly. 2012; 13706. doi:10.4414/smw.2012.13706.

19. Kohn LT, Corrigan JM, Donaldson MS. Institute of Medicine (US) Committee on Quality of Health Care in America. To err is human: building a safer health system, Washington (DC): National Academies Press (US) 2000. http:/ / www.ncbi.nlm. nih.gov/books/NBK225182/.Zugegriffen:22. Febr. 2017.

20. de Jonge V, Nicolaas JS, van Leerdam ME, Kuipers EJ. Overview of the quality assurance movement in health care. Best Pract Res Clin Gastroenterol. 2011;25:337-47.

21. Croskerry P. Perspectives on diagnostic failure and patient safety. Heal Q Tor Ont. 2012;15(sp):50-6.

22. Croskerry P. Achieving quality in clinical decision making: cognitive strategies and detection of bias. Acad Emerg Med. 2002;9:1184-204.

23. Kahneman D. Schnelles Denken, langsames Denken, 1. Aufl. München: Siedler; 2012. Aus dem Amerikan. Engl. von Thorsten Schmidt.

24. Marewski JN, Gigerenzer G. Heuristic decision making in medicine. Dialogues Clin Neurosci. 2012;14:77-89.

25. Waeschle RM, Bauer M, Schmidt CE. Fehler in der Medizin: Ursachen, Auswirkungen und Maßnahmen zur Verbesserung der Patientensicherheit. Anaesthesist. 2015;64:689-704.

26. Sox HC, Higgins MC, Owens DK. Medical decision making, 2. Aufl. Chichester: John Wiley \& Sons; 2013.

27. Kovacs G, Croskerry P. Clinical decision making: an emergency medicine perspective. Acad Emerg Med. 1999;6:947-52.

28. Croskerry P, Nimmo G. Better clinical decision making and reducing diagnostic error. J R Coll Physicians Edinb. 2011;41:155-62.

29. Croskerry P. Clinical cognition and diagnostic error: applications of a dual process model of reasoning. Adv Health Sci Educ. 2009; 14:27-35.

30. CroskerryP.Auniversalmodel of diagnostic reasoning.Acad Med. 2009;84:1022-8.

31. Wurmb T, Brederlau J. Patientenwille und Akutmedizin. Med Klin Intensivmed Notfallmed. 2016;111:113-7.

32. Hess EP, Grudzen CR, Thomson R, Raja AS, Carpenter CR. Shared decision-making in the emergency department: respecting patient autonomy when seconds count. Acad Emerg Med. 2015;22:856-64, Gaddis G, editor.

33. Jonsen AR, Siegler M, Winslade WJ. Clinical ethics: a practical approach to ethical decisions in clinical medicine, 8. Aufl. New York: McGraw-Hill Education; 2015.
34. Bioethikkommission. Empfehlungen zur Terminologie medizinischer Entscheidungen am Lebensende Stellungnahme der Bioethikkommission beim Bundeskanzleramt 2011. http://www.bundeskanzleramt.at/DocView.axd? CobId=46713.Zugegriffen: 9. Apr 2017.

35. Donabedian A. Evaluating the quality of medical care. Milbank Q. 2005;83:691-729, Reprinted from The Milbank Memorial Fund Quarterly, Vol. 44, No. 3, Pt. 2, 1966 (pp. 166-203).

36. Weick KE, Sutcliffe KM. Managing the unexpected: sustained performance in a complex world, 3. Aufl. Hoboken: Wiley; 2015.

37. Schulz CM, Endsley MR, Kochs EF, Gelb AW, Wagner KJ. Situation awareness in anesthesia: concept and research. Anesthesiology. 2013;118:729-42.

38. Vogel P, Vassilev G, Kruse B, Cankaya Y. Morbidity and mortality conference as part of PDCA cycle to decrease anastomoticfailurein colorectal surgery. Langenbecks Arch Surg. 2011;396:1009-15.

39. Xiong X, Johnson T, Jayaraman D, McDonald EG, Martel M, Barkun AN. At the crossroad with morbidity and mortality conferences: lessons learned through a narrative systematic review. Can J Gastroenterol Hepatol. 2016;2016:1-11. doi:10.1155/2016/7679196.

40. Bruno MA, Nagy P. Fundamentals of quality and safety in diagnostic radiology. J Am Coll Radiol. 2014;11:1115-20.

41. Reilly JB, Myers JS, Salvador D, Trowbridge RL. Use of a novel, modified fishbone diagram to analyze diagnostic errors. Diagnosis 2014. http://www.degruyter.com/view/ j/dx.2014.1.issue-2/dx-2013-0040/dx-2013-0040.xml. Zugegriffen: 19. Dec 2016.

42. Reason J. Human error: models and management. West J Med. 2000;172:393-6.

43. Landrigan CP, Rothschild JM, Cronin JW, Kaushal R, Burdick E, Katz JT, et al. Effect of reducing interns' work hours on serious medical errors in intensive care units. N Engl J Med. 2004;351:1838-48.

44. Nieva VF, Sorra J. Safety culture assessment: a tool for improving patient safety in healthcare organizations. Qual SafHealth Care. 2003;12(Suppl2):ii17-23.

45. Fleming M, Wentzell N. Patient safety culture improvement tool: development and guidelines for use. Healthc Q. 2008;11:10-5.

46. BerwickDM. Not again!BMJ.2001;322:247-8.

47. Croskerry P, Singhal G, Mamede S. Cognitive debiasing 1: origins of bias and theory of debiasing. BMJ Qual Saf. 2013;22:ii58-ii64.

48. Croskerry P, Singhal G, Mamede S. Cognitive debiasing 2: impediments to and strategies for change. BMJ Qual Saf. 2013;22:ii65-ii72.

49. Croskerry P. The importance of cognitive errors in diagnosis and strategies to minimize them. Acad Med. 2003;78:775-80.

50. Herold G. Innere Medizin. Köln: Gerd Herold Eigenverlag; 2012, S. 826-34. KapXI.

51. Henriksen K, Brady J. The pursuit of better diagnostic performance: a human factors perspective. BMJ Qual Saf. 2013;22:ii1-ii5.

52. El-Kareh R, Hasan O, Schiff GD. Use of health information technology to reduce diagnostic errors. BMJ Qual Saf. 2013;22:ii40-ii51

53. Schiff GD, Bates DW. Can electronic clinical documentation help prevent diagnostic errors? N Engl J Med. 2010;362:1066-9.

54. Gigerenzer G. Bauchentscheidungen: die Intelligenz des Unbewussten und die Macht der Intuition. München: Bertelsmann; 2015 . 


\section{übersicht}

55. Tsalatsanis A, Hozo I, Kumar A, Djulbegovic B. Dual processing model for medical decision-making: an extension to diagnostic testing. PLOS ONE. 2015;10:e0134800.

56. Clarke A. Ageism and age discrimination in primary and community health care in the United Kingdom. London: Centrefor Policy on Ageing; 2009.

57. Dale DC. Poor prognosis in elderly patients with cancer: the role of bias and undertreatment. J Support Oncol. 2003;1:11-7.
58. Peake MD, Thompson S, Lowe D, Pearson MG, et al. Ageism in the management of lung cancer. Age Ageing. 2003;32:171-7.

59. Eva KW. The aging physician: changes in cognitive processing and their impact on medical practice. Acad Med. 2002;77:S1-S6. 